\title{
THE STONE-ČECH COMPACTIFICATION AND WEAKLY FRECHET SPACES
}

\author{
Deborah M. King and Sidney A. Morris
}

This paper resulted from an attempt to answer questions like: does $\beta P$ have a subspace homeomorphic to $\beta Q$ and does $\beta Q$ have a subspace homeomorphic to $P$, where $P$ denotes the space of all irrational numbers. These questions are answered in the negative by providing the appropriate machinery which can also be applied to other examples. En route we prove that weakly Fréchet realcompact spaces have homeomorphic Stone-Čech compactifications if and only if they are homeomorphic.

NOTATION: If topological spaces $X$ and $Y$ are homeomorphic we write $X \cong Y$.

Definition: A topological space $Y$ is said to be a weakly Fréchet space if every point in $Y$ has a sequence of distinct points in $Y$ converging to it.

Some examples of weakly Fréchet spaces are: infinite first countable spaces with no isolated points, metrizable spaces with no isolated points, infinite Fréchet spaces with no isolated points, infinite path-connected spaces, infinite locally path-connected spaces, the product space $\prod_{i \in I} X_{i}$, where an infinite number of the $X_{i}$ have at least two points, and the product space $X \times Y$ where $X$ (or $Y$ ) is a weakly Fréchet space.

If $X$ is any Tychonoff space, then any continuous map of $X$ into $[0,1]$ can be extended (uniquely) to a continuous map of the Stone-Čech compactification of $X$, $\beta X$, into $[0,1]$. However, it is not true (for arbitrary $X$ ) that every continuous map of $X$ into $\mathbf{R}$ can be extended to a continuous map of $\beta X$ into $\mathbf{R}$. This leads to the notion of realcompactification of $X$.

Definition: Let $X$ be any Tychonoff space. Then the largest subspace $Y$ of $\beta X$ for which every continuous map into $\mathrm{R}$ can be extended to $Y$ is known as the (Hewitt) realcompactification of $X$, and is denoted by $v X$.

Definition: If $X$ is a Tychonoff space such that $v X=X$, then $X$ is said to be realcompact.

Received 22nd December 1989.

The authors wish to thank Wis Comfort, Karl Heinrich Hofmann, and Peter Nyikos for their helpful comments and encouragement.

Copyright Clearance Centre, Inc. Serial-fee code: 0004-9729/90 SA2.00+0.00. 
The class of realcompact spaces includes all separable metrizable spaces. (See $[2$, 8.5].)

Note that for any space $Z$ such that $X \subseteq Z \subseteq \beta X, \beta Z=\beta X$ (see $[2,6.7]$ ) and in particular $\beta(v X)=\beta X$ as $X \subseteq v X \subseteq \beta X$.

Theorem 1. Let $X$ be a Tychonoff space. If $y_{0} \in \beta X$ is such that there exists a sequence of distinct points $y_{n} \rightarrow y_{0}$, where $y_{n} \in \beta X$ for each $n \in N$, then $y_{0} \in v X$.

Proof: Suppose $y_{0} \in \beta X \backslash v X$ and $y_{n} \rightarrow y_{0}$ in $\beta X$, where $y_{n} \neq y_{0}$, for all $n \in \mathrm{N}$. Put $D=\left\{y_{n}: n \in \mathrm{N}\right\}$. Then $D$ is a discrete subspace of $\beta X$ with a limit point in $\beta X \backslash v X$. By [2, 9.10 and 9.4] $D$ contains a subspace $N$ homeomorphic to $\mathrm{N}$, such that $\operatorname{cl}_{\beta X} N=\beta N$. Observe that $\operatorname{cl}_{\beta X} N$ is uncountable as it is homeomorphic to the uncountable topological space $\beta N$. (See $[2,6.10]$.) As $D \cup\left\{y_{0}\right\}$ is closed in $\beta X$, we have that $D \cup\left\{y_{0}\right\}=\operatorname{cl}_{\beta X} D$ which has $\operatorname{cl}_{\beta X} N$ as a subspace. Therefore $D \cup\left\{y_{0}\right\}$ is uncountable, which is a contradiction. Hence $y_{0} \in v X$.

COROLlaRY 2. If $X$ is any Tychonoff space and $Y$ is a weakly Fréchet subspace of $\beta X$, then $Y \subseteq v X$.

COROLlary 3. If $X$ is any realcompact Tychonoff space and $Y$ is a weakly Fréchet subspace of $\beta X$, then $Y \subseteq X$.

EXAMPLE 4: Observe that $Q$ is a realcompact Tychonoff space and $P$, being a metrizable space with no isolated points, is a weakly Fréchet space. Suppose that $\boldsymbol{P}$ is homeomorphic to a subspace of $\beta Q$. Then, by Corollary $3, P$ would be homeomorphic to a subspace of $Q$. But $Q$ is countable while $P$ is uncountable. Hence, $P$ is not homeomorphic to a subspace of $\beta Q$.

Noting that $v X=\beta X$ if and only if $X$ is pseudocompact $([2,6 \mathrm{I}$ and $4,1.53])$, we obtain:

COROLlary 5. If $X$ is any Tychonoff space which is not pseudocompact, then $\beta X$ is not weakly Fréchet.

REMARK 6: The above result shows that, while the class of weakly Fréchet spaces is very large it certainly does not include all infinite compact Hausdorff spaces.

Corollary 7 . $[3,3.2]$ Let $X$ be a Tychonoff space and $f:[0,1] \rightarrow \beta X$ any non-constant path. Then $f([0,1]) \subseteq v X$.

Proof: This follows from Corollary 2 as $f([0,1])$ is weakly Fréchet.

COROLlary 8. If $X$ is a Tychonoff space such that $\beta X$ or $\beta X \backslash v X$ is pathconnected, then $X$ is pseudocompact.

COROLlaRY 9. Let $X$ be a normal realcompact space, $Y$ a weakly Fréchet space and $\beta Y \subseteq \beta X$. Then $Y \subseteq X$ and $\beta Y=\beta Z$, for $Z$ a closed subspace of $X$. 
Proof: Let $Z=\beta Y \cap X$. Then $Z$ is non-empty since $Y \subseteq X$ (by Corollary 2) and $Z$ is closed in $X$ since $\beta Y$ is closed in $\beta X$. But as $X$ is normal, Tietze's Extension Theorem implies that $\operatorname{cl}_{\beta X} Z=\beta Z$. Furthermore, $Y \subseteq Z \subseteq \beta Y$. Hence $\beta Z=\beta Y$.

REMARK 10: We might think that under the conditions of Corollary $9, Y$ would be a closed subspace of $X$. The following example shows this is not always true, while Proposition 11 shows that it is true when $X$ is metrizable.

Let $X$ be the disjoint union $[0,1] \cup \beta Q$ and let $Y=Q$. Then the space $Z=$ $\beta Q \cap([0,1] \cup \beta Q)=\beta Q$, and $\beta Z=\beta Q$, but $Q$ is not closed in $[0,1] \sqcup \beta Q$.

Proposition 11. Let $X$ be a metrizable realcompact topological space and $Y$ a topological space with $\beta Y \subseteq \beta X$. If $Y$ is metrizable with no isolated points then $Y$ is a closed subspace of $X$.

Proof: By Corollary 9, $\beta Y=\beta Z$, where $Z=\beta Y \cap X$. Since $Z$ is a closed subset of $X, Z$ is metrizable and so first countable. Thus by [2, p. 273], $Y=Z$. As $Z$ is closed in $X$, we have $Y$ is closed in $X$.

Corollary 12. Let $X$ be a separable metrizable space and $Y$ a metrizable space with no isolated points. Then $\beta Y$ is a subspace of $\beta X$ if and only if $Y$ is a closed subspace of $X$.

Proof: As $X$ is metrizable, it is a normal space. Therefore, if $Y$ is closed in $X$, then $\beta Y \subseteq \beta X$.

Conversely, assume that $\beta Y$ is a subspace of $\beta X$. As $X$ is separable metrizable, it is Hausdorff Lindelö, and thus realcompact [1, 3.11.12]. Therefore, by Proposition $11, Y$ is closed in $X$.

EXAMPLE 13: The topological space $\beta Q$ is not homeomorphic to a subspace of $\beta P$.

Proof: As $\mathbf{P}$ and $\mathbf{Q}$ are metrizable, if $\beta \mathbf{Q} \subseteq \beta \mathbf{P}$ then $\mathbf{P}$ would contain a closed copy of $Q$ (by Proposition 11). So it is enough to show that $P$ does not contain a closed copy of $\mathbf{Q}$. This is the case as $\mathbf{P}$ admits a complete metric (being a $G_{\delta}$ subset of $R$ ) while $Q$ does not (as it is not a $G_{\delta}$ subset of R.) (See $[1,4.3 .23]$ ).

It is well-known $[2$, p. 273] that first countable spaces which have homeomorphic Stone-Cech compactifications are themselves homeomorphic. As an extension of this result, we record the next two results.

Proposition 14. Let $X$ and $Y$ be weakly Fréchet Tychonoff spaces. Then $\beta X \cong \beta Y$ if and only if $v X \cong v Y$.

Proof: If $v X \cong v Y$, then $\beta(v X) \cong \beta(v Y)$. But $\beta(v X)=\beta X$ and $\beta(v Y)=\beta Y$. So $\beta X \cong \beta Y$. 
Conversely, assume $\beta X \cong \beta Y$. Without loss of generality we can assume that $\beta X=\beta Y$. As $Y$ is weakly Fréchet, $Y \subseteq v X$ by Corollary 2, and so $v Y \subseteq v X$. Similarly, $v X \subseteq v Y$. So $v X=v Y$.

COROLlary 15. Let $X$ and $Y$ be weakly Fréchet realcompact Tychonoff spaces. Then $\beta X \cong \beta Y$ if and only if $X \cong Y$.

\section{REFERENCES}

[1] R. Engelking, General Topology (Polish Scientific Publishers, Warsaw, 1977).

[2] L. Gillman and M. Jerison, Rings of Continuous Functions (Springer-Verlag, Berlin, Heidelberg, New York, 1960).

[3] R. Levy, 'Compactification by the topologists sine curve', Proc. Amer. Math. Soc. 63 (1977), 324-326.

[4] R.C. Walker, The Stone-Cech Compactification (Springer-Verlag, Berlin, Heidelberg, New York, 1974).

The University of New England

Armidale NSW 2351

Australia 